\title{
Correction to: An aptamer-based fluorometric zearalenone assay using a lighting-up silver nanocluster probe and catalyzed by a hairpin assembly
}

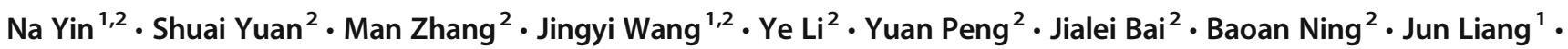 \\ Zhixian Gao ${ }^{2}$
}

Published online: 5 December 2019

(C) Springer-Verlag GmbH Austria, part of Springer Nature 2019

Correction to: Microchimica Acta (2019) 186:765

https://doi.org/10.1007/s00604-019-3984-6

The published version of this article contains an error. In manuscript and electronic supplementary material, there have to be two corresponding authors, viz. Jun Liang and Zhixian Gao. Given in this article are the correctly marked authors for correspondence.

Publisher's note Springer Nature remains neutral with regard to jurisdictional claims in published maps and institutional affiliations.

The online version of the original article can be found at https://doi.org/ 10.1007/s00604-019-3984-6

Jun Liang

jliang1118@yeah.net

$\triangle$ Zhixian Gao

gaozhx@163.com

1 State Key Laboratory of Food Nutrition and Safety, Tianjin University of Science and Technology, Tianjin 300457, China

2 Tianjin Key Laboratory of Risk Assessment and Control Technology for Environment and Food Safety, Tianjin Institute of Environmental and Operational Medicine, Tianjin 300050, China 